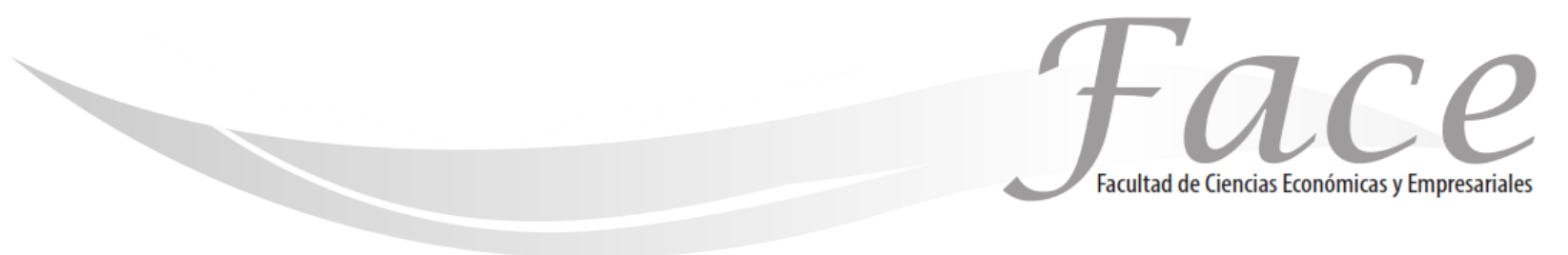

ISSN Impreso: 1794-9920

ISSN Electrónico: 2500-9338

Volumen $16-\mathrm{N}^{\circ} 2$

Año 2016

Págs. 18 - 27

\title{
FACTORES QUE INFLUYEN EN LA COMPETITIVIDAD: UN ANÁLISIS EMPÍRICO PARA LAS MICRO Y PEQUEÑAS EMPRESAS DEL MUNICIPIO DE GUADUAS*
}

\author{
Johana Regino Vergara ** \\ Enlace ORCID: http://orcid.org/0000-0002-7876-5957 \\ Sandra Milena Barbosa Castillo *** \\ Enlace ORCID: http://orcid.org/0000—0002-6626-3669
}

Fecha de Recepción: 7 de Septiembre 2016

Fecha de Aprobación: 14 de Diciembre 2016

\section{Resumen:}

Este artículo realiza un análisis empírico de los factores que influyen en la competitividad de las micro y pequeñas empresas del municipio de Guaduas- Colombia, mediante el modelo de análisis factorial exploratorio. Este permite la identificación de la oferta empresarial pertinente en la región y las estrategias más apropiadas para el fortalecimiento de los diferentes sectores económicos. Se evidencia desconocimiento de los nichos de mercado a los cuales se dirigen estas empresas y una gran fortaleza en la relación con sus proveedores, abriendo la posibilidad de contar con redes de cooperación entre las diferentes empresas.

Palabras Claves: Competitividad, Análisis factorial exploratorio, micro y pequeñas empresas, Desarrollo regional, Guaduas.

\footnotetext{
* Este artículo se deriva de la investigación terminada denominada "caracterización de las micro y pequeñas empresas del municipio de guaduas: un análisis empírico de los factores que influyen en la competitividad". Está vinculada a la interfaz de competitividad y desarrollo que maneja la Dirección General de Investigaciones de la Corporación Universitaria Minuto de Dios. El proyecto inició en el año 2015 y se encuentra finalizado.

${ }^{* *}$ Maestría en Dirección y Asesoramiento Financiero. Economista. Profesora Investigadora tiempo completo de la Facultad de Ciencias Empresariales, Corporación Universitaria Minuto de Dios, Colombia. Investigador principal del Semillero SECFIN (Semillero Económico, Contable y Financiero). Correo electrónico: jreginoverg@uniminuto.edu.co

***Especialista en Estadística. Profesora investigadora de la facultad de Ciencias Básicas, Corporación Universitaria Minuto de Dios, Colombia. Coinvestigadora del Semillero SECFIN. Correo electrónico: samibal74@gmail.com.
} 


\title{
FACTORS THAT INFLUENCE COMPETITIVENESS: AN EMPIRICAL ANALYSIS FOR MICRO AND SMALL ENTERPRISES IN THE MUNICIPALITY OF GUADUAS
}

\begin{abstract}
:
This article makes an empirical analysis of the factors that influence the competitiveness of micro and small enterprises in the municipality of Guaduas- Colombia, using the exploratory factor analysis model which allows to identify the relevant business offer in the region and the most appropriate strategies for strengthening the different economic sectors. There is a lack of knowledge of the market niches to which these companies are directed but there is also a great strength in the relationship with their suppliers, opening the possibility of having networks of cooperation between the different companies.
\end{abstract}

Keywords: Competitiveness, Exploratory factor analysis, micro and small enterprises, Regional development, Guaduas.

\section{FACTORES DE COMPETITIVIDADE: UMA ANÁLISE EMPÍRICA PARA MICRO E PEQUENAS EMPRESAS DO MUNICÍPIO DE GUADUAS}

\begin{abstract}
Resumo:
Este artigo faz uma análise empírica dos fatores que influenciam a competitividade das micro e pequenas empresas no município de Guaduas- Colombia, pelo modelo de análise fatorial exploratória. Isso permite identificação da empresa relevante na região e oferecer o mais adequado para o reforço das várias estratégias de sectores económicos, falta de nichos de mercado a que estas empresas e grande força na relação com o seu alvo fornecedores, abrindo a possibilidade de ter redes de cooperação entre empresas diferentes.
\end{abstract}

Palavras-Chave: Competitividade, análise fatorial exploratória, micro e pequenas empresas, desenvolvimento regional, Guaduas. 


\section{INTRODUCCIÓN:}

Para nadie es un secreto que Colombia cuenta con una dotación de recursos naturales y una localización estratégica que le permite lograr una posición de ventaja para lograr mayor competitividad y así enfrentar las exigencias de un entorno internacional cada vez más cambiante.

Sin embargo tiene una alta dependencia de los recursos naturales limitando su base exportadora. Es así como estudios de José Antonio Ocampo y la realizada por la cámara de comercio de Antioquia (2006), muestra que mientras el comercio nacional en volumen se ha incrementado la composición de las exportaciones prácticamente ha permanecido igual ( $p$ 109).

Eso significa que Colombia no ha entrado a competir en mercados internacionales con productos que generen un mayor valor agregado lo que limita el acceso a nuevos mercados y por ende la posibilidad de aumentar su oferta exportadora. Esto tiene un gran impacto en la competitividad del sector empresarial al no contar con un ambiente propicio para su desarrollo debido a las restricciones en su entorno.

Esta situación es aún más evidente a nivel regional, donde zonas como la provincia del Bajo Magdalena, que se caracterizan por ser de vocación agropecuaria, cuentan poco acceso a capital de trabajo y a tecnologías aplicadas que permita a los campesinos y agricultores resolver situaciones que se presentan en sus labores diarias. Esa falta de oportunidades limita la productividad y competitividad de las unidades familiares figura predominante en el sector empresarial de la región.

Esto se evidencia en el plan de competitividad de la provincia del bajo Magdalena donde la región representa solo el 2,8\% del mercado departamental consolidándose como el decimotercero de Cundinamarca y una de las provincias de menor dinamismo económico y empresarial.

Otra variable que impacta de manera significativa en la competitividad es la disponibilidad de la mano de obra calificada para el desarrollo de la región. En este aspecto cabe resaltar que la tendencia ha venido cambiando ya que la tasa de población de 25 a 39 años ha venido decreciendo lo cual se explica principalmente por el nivel de migración de la población en la región quienes se van a las ciudades por la falta de oportunidades que mejoren su calidad de vida.

A nivel de producción la provincia del Bajo Magdalena fue la tercera con menor contribución al departamento al representar solo el 2,4\% del Producto Interno Bruto. Este se concentra principalmente en el desarrollo de actividades agropecuarias $(25,2 \%)$ e industriales $(12,5 \%)$, y en menor medida construcción $(7,8 \%)$. Asimismo hacen un aporte significativo sectores como la administración pública $(9,3 \%)$, Minería $(8,2 \%)$ y los servicios financieros (CEPEC, 2011:25).

En ese sentido se hace necesaria la creación de un ambiente competitivo que fomente la inversión en factores estratégicos como el capital humano, procesos de innovación y una infraestructura moderna. Teniendo en cuenta lo anterior el análisis factorial tiene como objetivo la identificación de alianzas estratégicas que impulsen el desarrollo empresarial y económico de la provincia del Bajo Magdalena siendo el objeto de análisis inicialmente el municipio de Guaduas.

Asimismo surge la posibilidad de ofrecer nuevos servicios y productos en la región que propendan por el fortalecimiento y ampliación de la oferta de empleo el cual se garantiza mediante la conformación e inclusión en el mercado de empresas sostenibles acordes con las necesidades de la región.

\section{MARCO TEÓRICO:}

La competitividad es un concepto que aparece hace más de tres siglos y en el cual aún no se presenta un consenso acerca de su definición, así como los factores que lo componen. En la actualidad, se ha convertido en una prioridad de los entes gubernamentales y una de las principales causa de preocupación por parte de las empresas e inversionistas, quienes se han visto obligados a utilizar estrategias para lograr un mayor posicionamiento en el mercado y sostenibilidad a lo largo del tiempo.

Algunos autores han intentado plasmar diferentes definiciones de competitividad, relacionadas a factores como la productividad y la competencia internacional. Asimismo se le ha asociado con otro concepto como lo es la estrategia competitiva, la cual consiste en acciones llevadas a cabo por las empresas a través de la fijación de una serie de políticas y objetivos necesarios para poder competir (Quero, 2008).

Particularmente, Porter (1991:110) afirma que a nivel nacional este concepto está asociado de manera directa a la productividad, donde el objetivo principal es la generación de condiciones que contribuyan a mejorar la calidad de vida de los ciudadanos. Para este autor existen cuatro factores que afectan la competitividad del país y sus industrias: 
* Condiciones de los factores, que hacen referencia la posición de una región o nación con respecto a la dotación de los llamados factores especializados, los cuales en grandes cantidades, permiten alcanzar las ventajas competitivas. Estos son creados por cada país y responden a las necesidades de una industria concreta.

* Condiciones de la demanda, teniendo en cuenta que la calidad de la demanda local influye de manera significativa para el desarrollo de ventajas competitivas.

- Esta se consigue en aquellos sectores o territorios donde los compradores con mayor necesidad de productos $y$ servicios que se ajusten a las tendencias de los mercados internacionales, motivan a las empresas a que introduzcan de manera acelerada nuevos productos al mercado.

* Sectores afines y de apoyo, debido a que los proveedores locales de una región pueden contribuir a mejorar la competitividad, ya que permite la generación de "economías de aglomeración", aprovechando su cercanía geográfica y contribuye al fortalecimiento de los procesos productivos para las empresas de un determinado sector o territorio. Se obtiene la mayor ventaja cuando los proveedores son, a su vez, competidores a escala mundial.

* Estrategia, estructura y rivalidad de la empresa, relacionado a las condiciones vigentes de una región o nación respecto a las normas e incentivos que fomenten la inversión, faciliten la creación y gestión de las organizaciones, así como el grado de rivalidad de la competencia. Estas condiciones varían mucho de una nación a otra e incluso se evidencia en el contexto regional y local.
Es así como el aprovechamiento de estos factores se hace de manera abierta, haciendo uso de la información de fuentes externas tales como los proveedores, clientes e incluso la misma competencia ya que las empresas se benefician de usar las diferentes fuentes de información que más le permitan innovar y obtener ventajas competitivas para sostenerse en un mercado.

\section{METODOLOGÍA:}

El análisis de los factores que influyen en la competitividad del sector empresarial se realizó por medio de una investigación de carácter mixto por cuanto se usaron variables cualitativas de orden nominal, dicotómicas y cuantitativas discretas.

Se analizó la información a través de mapas factoriales al ser un modelo estadístico que tiene un gran impacto en cuanto a información analizada y sintetizada que facilita la toma de decisiones económicas para potencializar el crecimiento y desarrollo regional. Estadísticamente hablando el análisis factorial es de tipo exploratorio debido a que no se conocen a priori el número de variables, por lo que se reduce en un número determinado de factores sin perder información e interconectándolas entre sí.

Estos contribuyen a la identificación de la oferta empresarial pertinente en la región y las estrategias más apropiadas para el fortalecimiento de los diferentes sectores económicos mediante la cooperación entre las diferentes empresas.

La recolección de la información se realizó a través de una encuesta aplicada a 76 micro y pequeñas empresas del municipio de Guaduas por los estudiantes pertenecientes al semillero SECFIN de la Corporación Universitaria Minuto de Dios y la base de datos de la cámara de comercio de Honda siendo nuestra población objeto de estudio, debido a que es el tipo de empresa que predomina a nivel municipal y regional ${ }^{1}$. A continuación se muestra la ficha técnica de las encuestas aplicadas: 
ISSN: 1794-9920 - ISSN-E: 2500-9338

Volumen 16 Número: 2

Año: 2016. Junio - Diciembre - Págs. 18 - 27

Tabla $\mathbf{N}^{\circ} 1$.

Ficha técnica de la encuesta aplicada en las micro y pequeñas empresas del municipio de Guaduas

\begin{tabular}{cc}
\hline Zona de acción & Guaduas- Cundinamarca \\
\hline Universo/ Población & $\begin{array}{c}350 \text { micro y pequeñas empresas } \\
\text { registradas en la jurisdicción de la } \\
\text { cámara de comercio de Honda } \\
\text { durante el período 2014. }\end{array}$ \\
\hline Muestra & $\begin{array}{c}76 \text { micro y pequeñas empresas de } \\
\text { los diferentes sectores económicos } \\
\text { del municipio de Guaduas. }\end{array}$ \\
\hline Nivel de confianza & 95\% \\
\hline Aplicación & $\begin{array}{c}\text { En sitio. Los empresarios fueron } \\
\text { visitados por los estudiantes que } \\
\text { conforman el semillero de } \\
\text { investigación SECFIN. }\end{array}$ \\
\hline Fecha de aplicación & II semestre del 2015 \\
\hline Fuente: propia.
\end{tabular}

Fuente: propia.

Con respecto a la muestra que se relaciona en la Tabla 1 , el cálculo se detalla a continuación ${ }^{2}$ :

$$
\mathrm{n}=\frac{N \cdot Z^{2} \cdot p \cdot(1-p)}{(N-1) \cdot \mathrm{e}^{2}+Z^{2} \cdot p \cdot(1-p)}
$$

\section{N: 350}

Z: nivel de confianza de $95 \%$

p: 0,5 Son pequeñas y medianas empresas de guaduas de la zona comercial

1-p: 0,5 No son pequeñas y medianas empresas de guaduas de la zona comercial

N-1: 349

e: Error admitido que para nuestro caso es de 0,1

${ }^{2}$ Se utiliza este método de muestreo teniendo en cuenta que la población es conocida.

$$
\begin{array}{r}
n=\frac{350 *(1,96)^{2} * 0,5 * 0,5}{(350-1) *(0,1)^{2}+(1,96)^{2} * 0,5 * 0,5} \\
=75,53 \text { es decir } 76 \text { empresas. }
\end{array}
$$

En este sentido será posible validar el comportamiento de las unidades productivas identificando los factores que influyen de manera significativa en la productividad y establecer relaciones entre estos. La escala de medida y los factores escogidos se construyeron en base a la literatura descrita anteriormente. Por lo tanto, se identificaron variables de carácter cuantitativo como el volumen de ventas, productos base, egresos, número de empleados entre otras para analizar su contribución al desarrollo del sector empresarial y las relaciones existentes entre ellas

Se realizó un análisis factorial exploratorio en búsqueda de correlaciones entre variables que permitan identificar cargas factoriales y coeficientes que generen la reducción de las dimensiones. Es así como los factores cuyos residuos normalizados son elevados explican a aquellos cuyas cargas factoriales y coeficientes de determinación son menores a través de la reducción de dimensiones. Tras esa depuración se obtuvo un total de 5 factores que mostraron unos niveles de validez y fiabilidad superior a la propuesta inicialmente, por lo que procedimos a utilizarla para la estimación del modelo.

La consideración de escoger un modelo factorial se efectúa a través de un análisis exploratorio que evidencia la no normalidad de algunas variables por tanto se obtiene la matriz de correlaciones con el fin de validar relaciones entre las diferentes variables y obtener un determinante adecuado.

Por medio de La prueba de esfericidad de Bartlett y KMO se puede obtener estas correlaciones e interpretar la homogeneidad entre los factores para determinar la pertinencia de aplicar un análisis factorial de las características mencionadas anteriormente. En este sentido cuanto más se acerque a uno el indicador y la significancia sea inferior al $5 \%$ se valida la pertinencia del modelo.

Los valores obtenidos en la prueba de esfericidad de Bartlett y KMO mostraron unos niveles de validez adecuados, con un valor de significancia inferior al 0,05 con distribución chi-cuadrado y una prueba KMO mayor a 0,5 como se evidencia en la tabla 2 . Se rechaza la hipótesis nula, por lo tanto se acepta la realización de un análisis factorial. Esta hipótesis se validó a través del 
FACTORES QUE INFLUYEN EN LA COMPETITIVIDAD: UN ANÁLISIS EMPÍRICO PARA LAS MICRO Y PEQUEÑAS EMPRESAS DEL MUNICIPIO DE GUADUAS

Johana Regino Vergara - Sandra Milena Barbosa Castillo

programa SPSS 20 partiendo de los resultados que arrojaron las encuestas aplicadas.

Tabla $\mathbf{N}^{\circ} 2$.

Resultados prueba de esfericidad de Bartlett y KMO.

\begin{tabular}{crr}
\hline \multicolumn{3}{c}{$\begin{array}{c}\text { Medida de adecuación muestral de Kaiser-Meyer- } \\
\text { Olkin. }\end{array}$} \\
\hline \multirow{3}{*}{$\begin{array}{c}\text { Prueba de esfericidad de } \\
\text { Bartlett }\end{array}$} & Chi-cuadrado & 232,8 \\
& aproximado & 25 \\
\cline { 2 - 3 } & gl & 78 \\
\cline { 2 - 3 } & Sig. &, 000 \\
\hline
\end{tabular}

Fuente: propia.

Por medio del criterio de Kaiser se pueden obtener auto valores que nos permitan proceder a la reducción de dimensiones. Para el modelo planteado en párrafos anteriores, los valores que se ajusten por encima de 1 retienen y explican un valor importante de la varianza. Teniendo en cuenta los valores obtenidos en la tabla 3, solo 5 factores cumplen con la condición establecida con una varianza retenida del $53,55 \%$.
Fuente: propia. Se realizó por el método de extracción de Factorización Ejes principales.

Teniendo en cuenta lo anterior se obtiene un modelo en cinco factores que permite identificar las variables con mayor contribución en el posicionamiento de las empresas y por ende en el desarrollo de la región. También se evidencia la no relación e independencia de algunas variables, que pueden llegar a fortalecerse para lograr correlaciones que permitan mejorar la competitividad del sector empresarial en el municipio de Guaduas.

Finalmente se realiza el modelo factorial que contiene las correlaciones entre las variables originales y los cinco factores ya validados en el ítem anterior. El nivel mínimo de correlación para cada variable debe estar por encima de $60 \%$ para que tenga una contribución significativa dentro del factor asociado.

Tabla $\mathbf{N}^{\circ} 3$.

Escogencia de factores por varianza total explicada

\begin{tabular}{|c|c|c|c|c|c|c|}
\hline \multirow[t]{2}{*}{ Factor } & \multicolumn{3}{|c|}{ Autovalores iniciales } & \multicolumn{3}{|c|}{$\begin{array}{c}\text { Sumas de las saturaciones al cuadrado de la } \\
\text { extracción }\end{array}$} \\
\hline & Total & $\begin{array}{l}\% \text { de la } \\
\text { varianza }\end{array}$ & $\%$ acumulado & Total & $\begin{array}{l}\% \text { de la } \\
\text { varianza }\end{array}$ & $\%$ acumulado \\
\hline 1 & & 27,931 & 27,931 & 3,251 & 25,005 & 25,005 \\
\hline 2 & & 13,779 & 41,711 & 1,442 & 11,089 & 36,094 \\
\hline 3 & 1,520 & 11,694 & 53,405 & 941 & 7,238 & 43,332 \\
\hline 4 & 1,10 & 8,990 & 62,395 & 679 & 5,223 & 48,555 \\
\hline 5 & 1,040 & 8,000 & 70,395 & 648 & 4,982 & 53,537 \\
\hline 6 & 862 & 6,629 & 77,025 & & & \\
\hline 7 & 658 & 5,058 & 82,083 & & & \\
\hline 8 &, 625 & 4,805 & 86,887 & & & \\
\hline 9 &, 571 & 4,394 & 91,282 & & & \\
\hline 10 &, 454 & 3,493 & 94,774 & & & \\
\hline 11 &, 326 & 2,505 & 97,279 & & & \\
\hline 12 & ,229 & 1,763 & 99,042 & & & \\
\hline 13 &, 124 & ,958 & 100,000 & & & \\
\hline
\end{tabular}

Fuente: propia. 
ISSN: 1794-9920 - ISSN-E: 2500-9338

Volumen 16 Número: 2

Año: 2016. Junio - Diciembre - Págs. 18 - 27

Es así como estos se discriminan de la siguiente manera:

Primer factor: Está conformada por las variables de empleados fijos, empleados temporales, ventas al mes y clientes objetivos. Este factor evalúa los procesos relacionados a la segmentación de mercados y servicio al cliente.

Segundo Factor: Está compuesta por proveedores y tipo de ventas. En este factor se valoran aspectos relacionados con los canales de distribución y eficiencia en los procesos productivos.

Tercer Factor Se compone de clientes y tiempo de experiencia para identificar factores asociados al posicionamiento de las empresas y las estrategias para la captación de clientes.

Cuarto factor: Se compone de la naturaleza jurídica para evaluar aspectos asociados a la formalización de empresas y la aplicación del marco legal y tributario a nivel organizacional.

Quinto factor: Está formada por la variable de la clasificación de local donde se presta el servicio evaluando factores asociados a las diferentes alternativas de inversión y su destino.

Tabla $N^{\circ} 4$.

Matriz de Factores y su Composición

\begin{tabular}{|c|c|c|c|c|c|}
\hline & \multicolumn{5}{|c|}{ Factor } \\
\hline & 1 & 2 & 3 & 4 & 5 \\
\hline Naturaleza Jurídica & 180 &,- 415 & ,192 & 514 & ,266 \\
\hline Declara Renta &,- 657 &,- 082 &, 033 & 167 &, 046 \\
\hline Local &,- 036 &, 067 & ,258 &,- 190 &, 356 \\
\hline Empleados Fijos &, 500 &,- 198 &,- 274 &,- 106 &,- 012 \\
\hline Empleados Temporales & 815 &,- 194 &,- 008 & 155 &,- 075 \\
\hline Vinculación a Seguridad Social &,- 547 & ,380 & 049 & ,301 &,- 220 \\
\hline Tiene Socios &,- 565 & ,085 & 177 & 100 &,- 089 \\
\hline Clientes & ,178 &, 036 & 429 &,- 181 &,- 102 \\
\hline Proveedores son & 343 &, 782 &,- 158 & ,229 &, 426 \\
\hline Tiempo de Experiencia & ,115 & 129 & 653 &,- 152 & ,126 \\
\hline Ventas al mes & 888 &, 077 &, 033 &,- 012 &,- 026 \\
\hline Tipo de Ventas & 111 &, 590 &,- 107 &,- 149 &,- 165 \\
\hline Clientes Objetivo & 537 & ,212 & ,283 & 290 &,- 388 \\
\hline
\end{tabular}

Fuente: propia. Se realizó por el método de extracción de Factorización de ejes principales. 


\section{RESULTADOS:}

Teniendo en cuenta los resultados de la tabla 4 se pueden evidenciar diferentes situaciones que son importantes de resaltar para el fortalecimiento del tejido empresarial del municipio de Guaduas:

* Para el primer factor la variable de clientes objetivos tiene una baja contribución $(53,7 \%)$ lo que denota un desconocimiento del mercado al cual se dirigen y la débil aplicación de estrategias de mercado orientadas a mejorar la oferta de productos y servicios en el municipio.

* Para el segundo factor una variable que aporta de manera significativa es la relación con los proveedores $(78,2 \%)$ lo que puede influir en el desarrollo del sector empresarial debido a que cuenta con facilidades de acceso a materia prima e insumos necesarios para la implementación de procesos productivos que contribuyan a mejorar la competitividad en las empresas de la región.

* Para el tercer factor existe una gran debilidad en materia de Clientes (Solo tiene una contribución de $42,9 \%$ ) y aunque el tiempo de experiencia tiene una contribución por encima del mínimo aceptado $(65,3 \%)$ no es lo suficientemente fuerte, lo que implica que no cuentan con un buen posicionamiento en mercado y las estrategias para captar nuevos clientes no son suficientes para lograr altos niveles de rentabilidad lo que restringen sus posibilidades de crecimiento y expansión a nivel municipal y regional.

* Para el cuarto factor la variable naturaleza jurídica tiene una contribución por debajo del mínimo (51\%) lo que genera gran preocupación ya que son empresas compuestas en su gran mayoría por personas naturales que no se encuentran formalizadas ante la Cámara de Comercio lo que dificulta el acceso a beneficios y procesos de formación que permitan fortalecer las habilidades de negocio y el buen manejo de los recursos lo que significa un riesgo para ser sostenibles en el mercado.

* Asimismo se evidencia que aspectos legales como declarar de renta y admitir socios presentan muy baja correlación y no asociación con ninguna variable lo que permite corroborar la falta de capacitación en aspectos de carácter
* contable y tributario generando un gran riesgo de evasión y fragilidad en el sistema productivo del municipio.

* Para el quinto factor la clasificación del local donde se presta el servicio solo tiene un peso de $35 \%$ explicado por la dificultad de acceder a fuentes de financiación en el sector financiero lo que limita la realización de inversiones relacionadas con la adquisición de un local o la expansión del negocio afectando el desarrollo del sector empresarial y su competitividad.

\section{CONCLUSIONES:}

En este trabajo se ha propuesto una caracterización de las micro y pequeñas empresas del municipio de Guaduas examinando los diferentes factores que contribuyen al desarrollo de la provincia Bajo Magdalena y la competitividad en estas empresas. Se ha proporcionado evidencia empírica sobre la validez de este modelo y se han obtenido conclusiones relevantes para el fortalecimiento del tejido empresarial y factores para el éxito.

Como conclusiones principales se pueden mencionar las siguientes:

En primer lugar se demuestra el gran desconocimiento de los nichos de mercado a los cuales se dirigen estas empresas lo que limita sus posibilidades de crecimiento y expansión.

En segundo lugar se muestra una gran fortaleza en la relación con sus proveedores lo que es una gran ventaja para el desarrollo de sus procesos de producción y distribución al contar con las facilidades de acceso a materia prima e insumos necesarios generando posibilidad de contar con redes de cooperación entre las diferentes empresas.

En tercer lugar tienen grandes debilidades en materia de posicionamiento en el mercado y estrategias para atraer nuevos clientes lo que influye en los niveles de rentabilidad y crecimiento.

En cuarto lugar y uno de los factores más preocupantes es el gran vacío existente en los aspectos legales ya que son empresas que en su gran mayoría no están formalizadas, no declaran renta y la admisión de socios es débil lo que implica un sistema productivo frágil. 
En quinto lugar se presentan debilidades en materia de inversión debido a la dificultad de acceder a recursos por parte del sistema financiero afectando su competitividad.

Esto es coherente con la literatura en la medida en que son necesarios cambios significativos en estas empresas en las áreas de mercado, económica, contable y financiera así como generar condiciones que fomenten la competitividad y por consiguiente contribuyan al desarrollo de la provincia Bajo Magdalena fortaleciendo el tejido empresarial. Es así como se hace necesario el desarrollo de diferentes estrategias tales como estudios de carácter empírico así como procesos de asesorías a las empresas que les permitan enfrentar los retos en materia de recurso humano calificado, procesos de innovación e infraestructura moderna factores claves para la competitividad de las micro y pequeñas empresas del municipio de Guaduas.

\section{REFERENCIAS:}

Antúnez, A., Sanjuán, J. (2007). Análisis de Clúster en Andalucía. Investigaciones regionales. 12 (1). $79-106$.

Becerra, F., Álvarez, C. (2011). El talento humano y la innovación empresarial en el contexto de las redes empresariales: El clúster de prendas de vestir en Caldas Colombia. Estudios gerenciales. 27 (119). 209-232.

Cámara de Comercio de Medellín. (2006). Clúster, una estrategia para crear ventaja competitiva. Comunidad Clúster, Medellín y Antioquia. 1(1). 109.

Castro, F., Pedraza, O. (2013). El clúster forestal como estrategia de innovación para una empresa comunitaria exitosa. 8 (1). 13591367.

Centro de Pensamiento en Estrategias Competitivas. (2011). Plan de competitividad incluyente en la provincia del Bajo Magdalena. Recuperado de http://www.urosario.edu.co/urosario_fi les/17/17c4fee0-72f3-4f12-8fc7-

7dad857bc67f.pdf.

Colina, L., López, P. (1991). El análisis de componentes principales: Aplicación al análisis de datos secundarios. 37 (1). 31 -63.
Correa, J., Jaramillo, F. (2007). Una aproximación metodológica y prospectiva a la gestión financiera en las pequeñas empresas. Revista de Contaduría Universidad De Antioquia. 50 (1). 93-118.

Estrella, A ., Ruiz, J., Sánchez, M. (2012). ¿Cómo compiten las Pymes en los mercados internacionales? Análisis de un clúster local con vocación Exportadora. Investigaciones Europeas de Dirección y Economía de la Empresa. 18(1). 87-99.

García, J., Betancur, F. (2007). Una aproximación metodológica y prospectiva a la gestión financiera en las pequeñas empresas. Contaduría Universidad De Antioquia. (50). 93-118. Recuperado de http://aprendeenlinea.udea.edu.co/revi stas/index.php/cont/article/view/2123/ 1733.

Gremio de pymes crea programa para impulsar alianzas y elevar competitividad. [Recurso: Portafolio]. (2007, Oct 08). Noticias Financieras. Recuperado de http://www.portafolio.co/columnistas/l a-competitividad-opinion.

Harvard Business Review. (2007). La Ventaja Competitiva de las Naciones. 85 (11). 69-95. Recuperado de https://dialnet.unirioja.es/servlet/articulo?codi go $=2469800$.

Manzaneque, M., Vanegas, R., García, D. (2010). Diferentes procesos de fracaso empresarial: Un análisis dinámico a través de la aplicación de técnicas estadísticas clúster. 19 (1). 67-88.

Montoya, A., Castellanos, O. (2010). Situación de la competitividad de las pyme en Colombia: Elementos actuales y retos. Agronomía Colombiana. 28(1). 107-117. Recuperado de

http://search.proquest.com/docview/1 677585580 ? accountid $=48797$. 
Luján, C.,Olivas, J., Gonzales, H. (2013). Forestería comunitaria en México: modelo estratégico para empoderamiento y competitividad en la globalización. Revista Economía, Sociedad y Territorio. 49 (15). 665-696.

Quero, L. (2008). Estrategias competitivas: factor clave para el desarrollo. 10(4). 36-49. Recuperado http://www.revistanegotium.org.ve/pdf /10/Art3.pdf.

Soza, S. (2011). Encadenamientos y similitudes estructurales para las regiones de Chile. Revista de análisis económico. 26 (2). 81 110. 